\title{
Steiner Ratio for Riemannian Manifolds
}

\author{
D. Cieslik, A. O. Ivanov, A. A. Tuzhilin
}

\begin{abstract}
For a metric space $(X, \rho)$ and any finite subset $N \subset X$ by $\rho\left(\operatorname{SMT}_{N}\right)$ and $\rho\left(\mathrm{MST}_{N}\right)$ we denote respectively the lengths of a Steiner minimal tree and a minimal spanning tree with the boundary $N$. The Steiner ratio $m(X, \rho)$ of the metric space is the value $\inf _{\{N: N \subset X\}} \frac{\rho\left(\operatorname{SMT}_{N}\right)}{\rho\left(\mathrm{MST}_{N}\right)}$. In this paper we prove the following results describing the Steiner ratio of some manifolds:

(1) the Steiner ratio of an arbitrary $n$-dimensional connected Riemannian manifold $M$ does not exceed the Steiner ratio of $\mathbb{R}^{n}$;

(2) the Steiner ratio of the base of a locally isometric covering is more or equal than the Steiner ratio of the total space;

(3) the Steiner ratio of a flat two-dimensional torus, a flat Klein bottle, a projective plain having constant positive curvature is equal to $\sqrt{3} / 2$;

(4) the Steiner ratio of the curvature -1 Lobachevsky space does not exceed $3 / 4$;

(5) the Steiner ratio of an arbitrary surface of constant negative curvature -1 is strictly less than $\sqrt{3} / 2$.
\end{abstract}

Keywords: Steiner minimal tree (SMT), minimal spanning tree (MST), the Steiner problem, the Steiner ratio, metric space, Riemannian manifold.

\section{Introduction and main results}

Let $V$ be an arbitrary finite set. Recall that a graph $G$ on $V$ is the pair $(V, E)$, where $E$ is a finite set that consists of some pairs of elements from $V$. Notice that $E$ can contain several copies of some pair, and also $E$ can contain the pairs of the form $\{v, v\}$, where $v \in V$. Elements from $V$ are called vertices of $G$, and the elements from $E$ are called edges of $G$. The edges of the form $(v, v)$ are called loops, and if $E$ contains several copies of an edge $e=\left\{v, v^{\prime}\right\} \in E$, then the edge $e$ is called a multiple edge. For a given graph $G$ we denote the set of all its vertices by $V(G)$, and the set of all its edges by $E(G)$. For convenience, we shall often denote the edge $e=\{x, y\} \in E(G)$ by $x y$.

Sometimes it is useful to consider graphs as topological spaces glued from segments each of which corresponds to an edge of the graph. Such graphs are

A. Ivanov and A. Tuzhilin were partially supported by RFBR (grants 96-15-96142 and 98-01-00240) and INTAS (grant 97-0808). 
called topological graphs. A continuous mapping $\Gamma$ from a topological graph $G$ into a topological space is called a network; the topological graph $G$, and also the standard graph corresponding to $G$, are called the type of $\Gamma$ or the topology of $\Gamma$. Thus, the edges of a network are continuous curves in the ambient space. Moreover, all the terminology of the Topological Spaces Theory is transferred to the topological graphs and networks. If the ambient space is a smooth manifold, then a network in such space is called smooth (piecewise-smooth), if all its edges are smooth (piecewise-smooth).

A graph $G$ is called weighted if it is given a non-negative function $\omega: E(G) \rightarrow$ $\mathbb{R}$ called the weight function. The number $\omega(e)$ is called the weight of the edge $e \in E(G)$. The sum of the weights over all edges of $G$ is called the weight of the graph $G$ and it is denoted by $\omega(G)$. If $G$ is a connected weighted graph, then the set of all connected spanning subgraphs of $G$ having the least weight contains a tree. Each such tree is called a minimal spanning tree and is denoted by $\mathrm{MST}_{G}$. Notice that if all the weights are strictly greater than zero, then any connected spanning subgraph of $G$ of the least weight is a tree.

Let $X$ be a set, $\rho$ be a metric on $X$, and $N$ be an arbitrary finite subset of $X$. Let $G$ be a complete graph on $N$. The metric $\rho$ generates the weight function that assigns to each edge $x y \in E(G)$ the number $\rho(x, y)$. This weight function will be denoted by the same letter $\rho$. Minimal spanning tree in the graph $G$ is denoted by $\mathrm{MST}_{N}$. A minimal Steiner tree on the set $N$ or a minimal Steiner tree spanning the set $N$ is defined to be a tree $\Gamma, N \subset V(\Gamma)$, such that

$$
\rho(\Gamma)=\inf _{\{\bar{N}: \bar{N} \subset N\}} \rho\left(\operatorname{MST}_{\bar{N}}\right),
$$

where the least upper bound is taken over all finite subsets $\bar{N}$ in $X$ that contain $N$. A minimal Steiner tree on the set $N$ is denoted by $\mathrm{SMT}_{N}$.

Note that, generally speaking, an $\mathrm{SMT}_{N}$ exists not for any $N$ (one of the reasons of that can be the incompleteness of the metric space $(X, \rho)$ ). Nevertheless, the greatest lower bound from the definition of $\mathrm{SMT}_{N}$ does always exist. In what follows, the greatest lower bound from (1) is always denoted by $\rho\left(\mathrm{SMT}_{N}\right)$, irrespective of the existence of $\mathrm{SMT}_{N}$.

The novelty of Steiner's Problem is that new points, the Steiner points, may be introduced so that an interconnecting network of all these points will be shorter. Given a set of points, it is a priori unclear how many Steiner points one has to add in order to construct an SMT. Whereas Steiner's Problem is very hard as well in combinatorial as in computational sense, the determination of a Minimum Spanning Tree is simple. Consequently, we are interested in

Definition. The Steiner ratio $m(X, \rho)$ of a metric space $(X, \rho)$ is defined as the following value:

$$
m(X, \rho)=\inf _{\{N: N \subset X\}} \frac{\rho\left(\mathrm{SMT}_{N}\right)}{\rho\left(\mathrm{MST}_{N}\right)} .
$$

It is clear that the Steiner ratio of any metric space is always a nonnegative number with $m(X, \rho) \leq 1$. The Steiner ratio is a parameter of the considered 
space and describes the approximation ratio for Steiner's Problem. The quantity $m(X, \rho) \cdot \rho\left(\mathrm{MST}_{N}\right)$ would be a convenient lower bound for the length of an SMT for $N$ in $(X, \rho)$; that means, roughly speaking, $m(X, \rho)$ says how much the total length of an MST can be decreased by allowing Steiner points.

Proposition 1.1 (E.F.Moore, in [3]) For the Steiner ratio of any metric space $(X, \rho)$ the inequalities

$$
\frac{1}{2} \leq m(X, \rho) \leq 1
$$

hold.

It is also shown that these inequalities are the best possible ones over the class of metric spaces 1

As an introductory example consider three points which form the nodes of an equilateral triangle of unit side length in the Euclidean plane. An MST for these points has length 2. An SMT uses one Steiner point, which is uniquely determined by the condition that the three angles at this point are equal, and consequently equal $120^{\circ}$. Consequently, we find the length of the SMT in 3 . $\sqrt{1 / 3}=\sqrt{3}$. So we have an upper bound for the Steiner ratio of the Euclidean plane:

$$
m \leq \frac{\sqrt{3}}{2}=0.86602 \ldots
$$

A long-standing conjecture, given by Gilbert and Pollak $[3$ in 1968, said that in the above inequality equality holds. This was the most important conjecture in the area of Steiner's Problem in the following years. Finally, in 1990, Du and Hwang 2] created many new methods and succeeded in proving the GilbertPollak conjecture completely: The Steiner Ratio of the Euclidean plane equals $\sqrt{3} / 2=0.86602 \ldots .2$

For each dimension $n>2$, at present, exact values for the Steiner ratios of the Euclidean spaces are not yet known. In particular, this is true for $n=3$.

SMT's have been the subject of extensive investigations during the past 30 years or so. Most of this research has dealt with the Euclidean metric, with much of the remaining work concerned with the $\mathcal{L}_{1}$-metric, or more generally, the usual $\mathcal{L}_{p}$-metric or with two-dimensional Banach spaces. An overview for the Steiner ratios of these metric spaces is given in 1 .

The first results concerning the Steiner ratios of Riemannian manifolds different from Euclidean spaces were obtained by J. H. Rubinstein and J. F. Weng in 1997, see [7. They have shown that the Steiner ratio for the standard twodimensional spheres is the same as for the Euclidean plane, that is, $\sqrt{3} / 2$.

Now we list the main results of the present article. These results were obtained by means of the technique worked out in [1, [5], and [6]. Let us mention that in [5] and [6] the authors investigate so called local minimal networks which turn out to be useful in the subject.

\footnotetext{
${ }^{1}$ And, indeed, there are metric spaces with Steiner ratios equals 1 and equals 0.5.

${ }^{2}$ This mathematical fact went in The New York Times, October 30, 1990 under the title "Solution to Old Puzzle: How Short a Shortcut?"
} 
Theorem 1.1 The Steiner ratio of an arbitrary n-dimensional connected Riemannian manifold $M$ does not exceed the Steiner ratio of $\mathbb{R}^{n}$.

Theorem 1.2 Let $\pi: W \rightarrow M$ be a locally isometric covering of connected Riemannian manifolds. Then the Steiner ratio of the base $M$ of the covering is more or equal than the Steiner ratio of the total space $W$.

Corollary 1.1 The Steiner ratio for a flat two-dimensional torus, a flat Klein bottle, a projective plain having constant positive curvature is equal to $\sqrt{3} / 2$.

Thus, taking into account the results of J. H. Rubinstein and J. F. Weng [7, the Steiner ratio is computed now for all closed surfaces having non-negative curvature.

Theorem 1.3 The Steiner ratio of the curvature -1 Lobachevsky space does not exceed $3 / 4$.

Theorem 1.4 The Steiner ratio of an arbitrary surface of constant negative curvature -1 is strictly less than $\sqrt{3} / 2$.

The authors want to thank the Ernst-Moritz-Arndt University of Greifswald for the opportunity to work together in Greifswald in March 2000. A. Ivanov and A. Tuzhilin are grateful to academic A. T. Fomenko for his kind interest to our work.

\section{Proofs of the theorems}

In the present section we give the proofs of the theorems stated above.

We need the following two Lemmas proved in [1] (notice that Lemma 2.1] is proved in [1] for the case of normalized spaces only, but the proof in the general case of metric spaces is just the same.)

Lemma 2.1 Let $X$ be a set, and $\rho_{1}$ and $\rho_{2}$ be two metrics on $X$. We assume that for some numbers $c_{2} \geq c_{1}>0$ and for arbitrary points $x$ and $y$ from $X$ the following inequality holds: $c_{1} \rho_{2}(x, y) \leq \rho_{1}(x, y) \leq c_{2} \rho_{2}(x, y)$. Then

$$
\frac{c_{1}}{c_{2}} m\left(X, \rho_{2}\right) \leq m\left(X, \rho_{1}\right) \leq \frac{c_{2}}{c_{1}} m\left(X, \rho_{2}\right) .
$$

Lemma 2.2 Let $(X, \rho)$ be a metric space, and $Y \subset X$ be some its subspace. Then

$$
m(Y, \rho) \geq m(X, \rho) .
$$

The following Proposition holds. 
Proposition 2.1 Let $f: X \rightarrow Y$ be some mapping of a metric space $\left(X, \rho_{X}\right)$ onto a metric space $\left(Y, \rho_{Y}\right)$. We assume that $f$ does not increase the distances, that is, for arbitrary points $x$ and $y$ from $X$ the following inequality holds:

$$
\rho_{Y}(f(x), f(y)) \leq \rho_{X}(x, y) .
$$

Then for arbitrary finite set $N \subset Y$ we have:

$$
\rho_{X}\left(\operatorname{MST}_{N}\right) \geq \rho_{Y}\left(\operatorname{MST}_{f(N)}\right), \quad \rho_{X}\left(\operatorname{SMT}_{N}\right) \geq \rho_{Y}\left(\operatorname{SMT}_{f(N)}\right) .
$$

Proof. Let $G$ be an arbitrary connected graph constructed on $N$. We consider two weight functions on $G$ defined on the edges $x y$ of $G$ as follows: $\rho_{X}(x y)=$ $\rho_{X}(x, y)$, and $\omega_{Y}(x y)=\rho_{Y}(f(x), f(y))$. Since $f$ does not increase the distances, then $\rho_{X}(G) \geq \omega_{Y}(G)$.

Let $G^{\prime}$ be a graph on $N^{\prime}=f(N)$, such that the number of edges joining the vertices $x^{\prime}$ and $y^{\prime}$ from $N^{\prime}=V\left(G^{\prime}\right)$ is equal to the number of edges from $G$ joining the vertices from $f^{-1}\left(x^{\prime}\right) \cap N$ with the vertices from $f^{-1}\left(y^{\prime}\right) \cap N$. It is clear that $G^{\prime}$ is connected, and $\rho_{Y}\left(G^{\prime}\right)=\omega_{Y}(G)$.

Conversely, it is easy to see that for an arbitrary connected graph $G^{\prime}$ constructed on $f(N)$ there exists a connected graph $G_{X}$ on $N$, such that $\rho_{Y}\left(G^{\prime}\right)=$ $\omega_{Y}\left(G_{X}\right)$. (To construct $G_{X}$ it suffices to span each set $N \cap f^{-1}\left(x^{\prime}\right), x^{\prime} \in N^{\prime}$, by a connected graph, and then to join each pair of the constructed graphs corresponding to some adjacent vertices $G^{\prime}$ by $k$ edges, where $k$ is the multiplicity of the corresponding edge in $G^{\prime}$ ). Therefore,

$$
\begin{aligned}
\rho_{X}\left(\operatorname{MST}_{N}\right)=\inf _{\{G: V(G)=N\}} \rho_{X}(G) \geq & \inf _{\{G: V(G)=N\}} \omega_{Y}(G)= \\
& \inf _{\left\{G^{\prime}: V\left(G^{\prime}\right)=f(N)\right\}} \rho_{Y}\left(G^{\prime}\right)=\rho_{Y}\left(\operatorname{MST}_{f(N)}\right) .
\end{aligned}
$$

Thereby, the first inequality is proved.

Now let us prove the second inequality. We have:

$$
\begin{aligned}
\rho_{X}\left(\mathrm{SMT}_{N}\right)= & \inf _{\{\bar{N}: \bar{N} \supset N\}} \rho_{X}\left(\mathrm{MST}_{\bar{N}}\right) \geq \inf _{\{\bar{N}: \bar{N} \supset N\}} \rho_{Y}\left(\operatorname{MST}_{f(\bar{N})}\right) \geq \\
& \inf _{\left\{\bar{N}^{\prime}: \bar{N}^{\prime} \supset f(N)\right\}} \rho_{Y}\left(\mathrm{MST}_{\bar{N}^{\prime}}\right)=\rho_{Y}\left(\mathrm{SMT}_{f(N)}\right) .
\end{aligned}
$$

The proof is complete.

Proposition 2.2 Let $f: X \rightarrow Y$ be a mapping of a metric space $\left(X, \rho_{X}\right)$ to a metric space $\left(Y, \rho_{Y}\right)$, and let $f$ do not increase the distances. We assume that for each finite subset $N^{\prime} \subset Y$ there exists a finite subset $N \subset X$, such that $f(N)=N^{\prime}$ and

$$
\rho_{X}\left(\mathrm{SMT}_{N}\right) \leq \rho_{Y}\left(\mathrm{SMT}_{N^{\prime}}\right)
$$

Then

$$
m\left(X, \rho_{X}\right) \leq m\left(Y, \rho_{Y}\right)
$$


Proof. Let $N \subset X$ be an arbitrary finite set. We have

$$
\begin{aligned}
m\left(X, \rho_{X}\right)= & \inf _{\{N: N \subset X\}} \frac{\rho_{X}\left(\mathrm{SMT}_{N}\right)}{\rho_{X}\left(\mathrm{MST}_{N}\right)}= \\
& \inf _{\left\{N^{\prime}: N^{\prime} \subset Y\right\}\left\{N: f(N)=N^{\prime}\right\}} \frac{\rho_{X}\left(\mathrm{SMT}_{N}\right)}{\rho_{X}\left(\mathrm{MST}_{N}\right)} \leq \\
& \inf _{\left\{N^{\prime}: N^{\prime} \subset Y\right\}} \frac{\rho_{Y}\left(\mathrm{SMT}_{N^{\prime}}\right)}{\rho_{Y}\left(\mathrm{MST}_{N^{\prime}}\right)}=m\left(Y, \rho_{Y}\right),
\end{aligned}
$$

where the inequality follows from both condition (3) and the first inequality of Proposition 2.1. The proof is complete.

Proposition 2.2 can be slightly reinforced as follows.

Proposition 2.3 Let $f: X \rightarrow Y$ be a mapping of a metric space $\left(X, \rho_{X}\right)$ to a metric space $\left(Y, \rho_{Y}\right)$, and let $f$ do not increase the distances. We assume that for each finite subset $N^{\prime} \subset Y$ the following inequality holds:

$$
\inf _{\left\{N: f(N)=N^{\prime}\right\}} \rho_{X}\left(\mathrm{SMT}_{N}\right) \leq \rho_{Y}\left(\mathrm{SMT}_{N^{\prime}}\right) .
$$

Then

$$
m\left(X, \rho_{X}\right) \leq m\left(Y, \rho_{Y}\right) .
$$

Proof. Let $N \subset X$ be an arbitrary finite set. As in the proof of Proposition 2.2 we have:

$$
m\left(X, \rho_{X}\right)=\inf _{\{N: N \subset X\}} \frac{\rho_{X}\left(\mathrm{SMT}_{N}\right)}{\rho_{X}\left(\mathrm{MST}_{N}\right)}=\inf _{\left\{N^{\prime}: N^{\prime} \subset Y\right\}} \inf _{\left\{N: f(N)=N^{\prime}\right\}} \frac{\rho_{X}\left(\mathrm{SMT}_{N}\right)}{\rho_{X}\left(\mathrm{MST}_{N}\right)} .
$$

Since $f$ does not increase distances, then $\rho_{X}\left(\operatorname{MST}_{N}\right) \geq \rho_{Y}\left(\operatorname{MST}_{f(N)}\right)$ (see Proposition 2.1); on the other hand, due to our assumption, there exists a sequence of finite sets $N_{i} \subset X, f\left(N_{i}\right)=N^{\prime}$, such that $\rho_{X}\left(\mathrm{SMT}_{N_{i}}\right) \leq \rho_{Y}\left(\mathrm{SMT}_{N^{\prime}}\right)+$ $\varepsilon_{i}$, where the sequence of positive numbers $\varepsilon_{i}$ tends to 0 as $i \rightarrow \infty$, and the se-

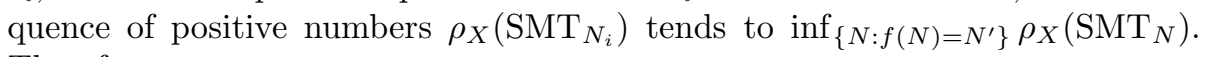
Therefore,

$$
\frac{\rho_{X}\left(\mathrm{SMT}_{N_{i}}\right)}{\rho_{X}\left(\mathrm{MST}_{N_{i}}\right)} \leq \frac{\rho_{Y}\left(\mathrm{SMT}_{N^{\prime}}\right)+\varepsilon_{i}}{\rho_{Y}\left(\mathrm{MST}_{N^{\prime}}\right)}
$$

and, taking in account that $\left\{N_{i}\right\} \subset\left\{N: f(N)=N^{\prime}\right\}$, we get:

$$
\begin{aligned}
\inf _{\left\{N^{\prime}: N^{\prime} \subset Y\right\}} \inf _{\left\{N: f(N)=N^{\prime}\right\}} \frac{\rho_{X}\left(\mathrm{SMT}_{N}\right)}{\rho_{X}\left(\mathrm{MST}_{N}\right)} \leq & \\
\inf _{\left\{N^{\prime}: N^{\prime} \subset Y\right\}} \inf _{\left\{N_{i}\right\}} \frac{\rho_{X}\left(\mathrm{SMT}_{N_{i}}\right)}{\rho_{X}\left(\mathrm{MST}_{N_{i}}\right)} \leq & \inf _{\left\{N^{\prime}: N^{\prime} \subset Y\right\}} \inf _{i} \frac{\rho_{Y}\left(\mathrm{SMT}_{N^{\prime}}\right)+\varepsilon_{i}}{\rho_{Y}\left(\mathrm{MST}_{N^{\prime}}\right)}= \\
& \inf _{\left\{N^{\prime}: N^{\prime} \subset Y\right\}} \frac{\rho_{Y}\left(\mathrm{SMT}_{N^{\prime}}\right)}{\rho_{Y}\left(\mathrm{MST}_{N^{\prime}}\right)}=m\left(Y, \rho_{Y}\right) .
\end{aligned}
$$

The proof is complete. 
Let $M$ be an arbitrary connected $n$-dimensional Riemannian manifold. For each piecewise-smooth curve $\gamma$ by len $(\gamma)$ we denote the length of $\gamma$ with respect to the Riemannian metric. By $\rho$ we denote the intrinsic metric generated by the Riemannian metric. We recall that

$$
\rho(x, y)=\inf _{\gamma} \operatorname{len}(\gamma)
$$

where the greatest lower bound is taken over all piecewise-smooth curves $\gamma$ joining the points $x$ and $y$.

Let $P$ be a point from $M$. We consider the normal coordinates $\left(x^{1}, \ldots, x^{n}\right)$ centered at $P$, such that the Riemannian metric $g_{i j}(x)$ calculated at $P$ coincides with $\delta_{i j}$. Let $U(\delta)$ be the open convex ball centered at $P$ and having the radius $\delta$. Any two points $x$ and $y$ from the ball are joined by a unique geodesic $\gamma$ lying in $U(\delta)$. At that time, $\rho(x, y)=\operatorname{len}(\gamma)$. Thus, the ball $U(\delta)$ is a metric space with intrinsic metric, that is, the distance between the points equals to the greatest lower bound of the curves' lengths over all the measurable curves joining the points. Notice that in terms of the coordinates $\left(x^{i}\right)$ the ball $U(\delta)$ is defined as follows:

$$
U(\delta)=\left\{\left(x^{1}\right)^{2}+\cdots+\left(x^{n}\right)^{2}<\delta^{2}\right\} .
$$

Therefore, if we define the Euclidean distance $\rho_{e}$ in $U(\delta)$ (in terms of the normal coordinates $\left.\left(x^{i}\right)\right)$, then the metric space $\left(U(\delta), \rho_{e}\right)$ also is the space with intrinsic metric generated by the Euclidean metric $\delta_{i j}$.

Since the Riemannian metric $g_{i j}(x)$ depends on $x \in U(\varepsilon)$ smoothly, then for any $\varepsilon, 1 / n^{2}>\varepsilon>0$, there exists a $\delta>0$, such that

$$
\left|g_{i j}(x)-\delta_{i j}\right|<\varepsilon
$$

for all points $x \in U(\delta)$. The latter implies the following Proposition.

Proposition 2.4 Let $\|v\|_{g}$ be the length of the tangent vector $v \in T_{x} M$ with respect to the Riemannian metric $g_{i j}$, and let $\|v\|_{e}$ be the length of $v$ with respect to the Euclidean metric $\delta_{i j}$. If for any $i$ and $j$ the inequality (5) holds, then

$$
\sqrt{1-n^{2} \varepsilon}\|v\|_{e} \leq\|v\|_{g} \leq \sqrt{1+n^{2} \varepsilon}\|v\|_{e} .
$$

Proof. Consider an orthogonal transformation (with respect to the Euclidean metric $\left.\delta_{i j}\right)$ reducing the matrix $\left(g_{i j}\right)$ to the diagonal form $\operatorname{diag}\left(\lambda_{1}, \ldots, \lambda_{n}\right)$, and let $\left(c_{j}^{i}\right)$ be the matrix of this transformation. Then $\lambda_{k}=\sum_{i, j} c_{k}^{i} c_{k}^{j} g_{i j}$, therefore, using that $\left|c_{j}^{i}\right| \leq 1$ due to orthogonality of $\left(c_{j}^{i}\right)$, we get:

$$
\begin{aligned}
\left|\lambda_{k}-1\right|=\left|\sum_{i, j}\left(c_{k}^{i} c_{k}^{j} g_{i j}-c_{k}^{i} c_{k}^{j} \delta_{i j}\right)\right| \leq \\
\sum_{i, j}\left|c_{k}^{i}\right| \cdot\left|c_{k}^{j}\right| \cdot\left|g_{i j}-\delta_{i j}\right| \leq \sum_{i, j}\left|g_{i j}-\delta_{i j}\right| \leq n^{2} \varepsilon .
\end{aligned}
$$


So we have:

$$
\|v\|_{g}=\sqrt{\sum_{k} \lambda_{k} v^{k} v^{k}} \leq \sqrt{\max _{k} \lambda_{k} \sum_{k} v^{k} v^{k}} \leq \sqrt{1+n^{2} \varepsilon}\|v\|_{e} .
$$

Similarly, we get

$$
\|v\|_{g} \geq \sqrt{1-n^{2} \varepsilon}\|v\|_{e}
$$

The proof is complete.

Using the definition of the distance between a pair of points of a connected Riemannian manifold, we obtain the following result.

Corollary 2.1 Let $M$ be an arbitrary connected n-dimensional Riemannian manifold, and let $U(\delta), \rho$, and $\rho_{e}$ be as above. Then for an arbitrary $\varepsilon, 1 / n^{2}>$ $\varepsilon>0$, there exists a $\delta>0$, such that

$$
\sqrt{1-n^{2} \varepsilon} \rho_{e}(x, y) \leq \rho(x, y) \leq \sqrt{1+n^{2} \varepsilon} \rho_{e}(x, y)
$$

for all points $x, y \in U(\delta)$.

Since the Steiner ratio is evidently the same for any convex open subsets of $\mathbb{R}^{n}$, Corollary 2.1 and Lemma 2.1 lead to the following result.

Corollary 2.2 Let $M$ be an arbitrary $n$-dimensional Riemannian manifold, let $U(\varepsilon) \subset M$ be an open convex ball of a small radius $\varepsilon$, and let $P$ be the center of $U(\varepsilon)$. By $\rho$ we denote the metric on $M$ generated by the Riemannian metric.

Then

$$
\sqrt{\frac{1-n^{2} \varepsilon}{1+n^{2} \varepsilon}} m\left(\mathbb{R}^{n}\right) \leq m(U(\varepsilon), \rho) \leq \sqrt{\frac{1+n^{2} \varepsilon}{1-n^{2} \varepsilon}} m\left(\mathbb{R}^{n}\right),
$$

where $m\left(\mathbb{R}^{n}\right)$ stands for the Steiner ratio of the Euclidean space $\mathbb{R}^{n}$.

Now let us prove the main theorems stated in Introduction.

Proof of Theorem 1.1. Let $M$ be an arbitrary connected $n$-dimensional Riemannian manifold, and let $\rho$ be the metric generated by the Riemannian metric of $M$. Let $P \in M$ be an arbitrary point from $M$, and let $U(\varepsilon)$ be an open convex ball centered at $P$ and having radius $\varepsilon<1 / n^{2}$. As above, let $\left(x^{i}\right)$ be normal coordinates on $U(\varepsilon)$, and let $\rho_{e}$ be the metric on $U(\varepsilon)$ generated by the Euclidean metric $\delta_{i j}$ (with respect to $\left(x^{i}\right)$ ).

For some decreasing sequence $\left\{\varepsilon_{i}\right\}$ of positive numbers with $\varepsilon_{i}<\varepsilon$ for any $i$, where $\varepsilon_{i} \rightarrow 0$ as $i \rightarrow \infty$, we consider a family of nested subsets $X_{i}=U\left(\varepsilon_{i}\right)$. Notice that due to convexity of Euclidean balls $\left(U(\varepsilon), \rho_{e}\right)$ we have:

$$
m\left(U(\varepsilon), \rho_{e}\right)=m\left(\mathbb{R}^{n}\right) .
$$

Besides, due to convexity of the balls $U(\varepsilon)$ with respect to the intrinsic metric $\rho^{\prime}$ generated by the Riemannian metric $g_{i j}$, this intrinsic metric $\rho^{\prime}$ coincides with 
the restriction of the metric $\rho$. Thus, the ball $U(\varepsilon)$ with the intrinsic metric $\rho^{\prime}$ is a subspace in $(M, \rho)$.

Corollary 2.2 implies that

$$
m\left(X_{i}, \rho\right) \leq \sqrt{\frac{1+n^{2} \varepsilon}{1-n^{2} \varepsilon}} m\left(\mathbb{R}^{n}\right) .
$$

Since $\sqrt{\frac{1+n^{2} \varepsilon}{1-n^{2} \varepsilon}} \rightarrow 1$ as $i \rightarrow \infty$ due to the choice of $\left\{\varepsilon_{i}\right\}$, we get

$$
\inf _{i} m\left(X_{i}, \rho\right) \leq m\left(\mathbb{R}^{n}\right) .
$$

But, due to Lemma 2.2 we have:

$$
m(M, \rho) \leq \inf _{i} m\left(X_{i}, \rho\right) .
$$

The proof is complete.

Proof of Theorem 1.2, Let $\pi: W \rightarrow M$ be a locally isometric covering, where $W$ and $M$ are connected Riemannian manifolds. By $\rho_{W}$ and $\rho_{M}$ we denote the metrics generated by the Riemannian metrics on $W$ and $M$, respectively. Notice that a locally isometric covering does not increase distances, since the image of a measurable curve $\gamma$ has the same length as $\gamma$ has.

We consider an arbitrary finite set $N^{\prime} \subset M$. Let $G_{i}^{\prime}$ be a family of trees on finite sets $\bar{N}_{i}^{\prime} \supset N^{\prime}$ such that

$$
\rho_{M}\left(G_{i}^{\prime}\right) \rightarrow \rho_{M}\left(\mathrm{SMT}_{N^{\prime}}\right) \text { as } i \rightarrow \infty .
$$

For each $G_{i}^{\prime}$ by $\Gamma_{i}^{\prime}$ we denote an embedded network of the type $G_{i}^{\prime}$ on $M$ such that the vertex set of $\Gamma_{i}^{\prime}$ is $V\left(G_{i}^{\prime}\right)$ and the length of $\Gamma_{i}^{\prime}$ differs from $\rho_{M}\left(G_{i}^{\prime}\right)$ at most by $1 / i$. Let $\Gamma_{i}$ be a connected component of $\pi^{-1}\left(\Gamma_{i}^{\prime}\right)$, and $N_{i}=\pi^{-1}(N) \cap \Gamma_{i}$. Since the network $\Gamma_{i}^{\prime}$ is contractible, then the restriction of the fibration $\pi$ onto $\Gamma_{i}^{\prime}$ is trivial. Therefore the restriction of the projection $\pi$ onto $\Gamma_{i}$ is a homeomorphism. Since the projection $\pi$ is locally isometric, then the length of the network $\Gamma_{i}$ in $W$ coincides with the length of the network $\Gamma_{i}^{\prime}$ in $M$. But $\rho_{W}\left(\mathrm{SMT}_{N_{i}}\right)$ does not exceed the length of $\Gamma_{i}$, therefore

$$
\rho_{W}\left(\operatorname{SMT}_{N_{i}}\right) \leq \rho_{M}\left(\operatorname{SMT}_{N^{\prime}}\right)+\varepsilon_{i},
$$

where the sequence $\left\{\varepsilon_{i}\right\}$ of positive numbers tends to 0 as $i \rightarrow \infty$. So,

$$
\inf _{\left\{N: f(N)=N^{\prime}\right\}} \rho_{W}\left(\mathrm{SMT}_{N}\right) \leq \rho_{M}\left(\mathrm{SMT}_{N^{\prime}}\right) .
$$

It remains to apply Proposition 2.3. The proof is complete.

Proof of Corollary 1.1, It follows from Theorems 1.1, and 1.2, Du and Hwang theorem [2] saying that the Steiner ratio of the Euclidean plane equals $\sqrt{3} / 2$; and also from Rubinstein and Weng theorem [7] saying that the Steiner ratio of the standard two dimensional sphere with constant positive curvature metric equals $\sqrt{3} / 2$. 
Proof of Theorem 1.3. Let us consider the Poincaré model of the Lobachevsky plane $L^{2}(-1)$ with constant curvature -1 . We recall that this model is a radius 1 flat disk centered at the origin of the Euclidean plane with Cartesian coordinates $(x, y)$, and the metric $d s^{2}$ in the disk is defined as follows:

$$
d s^{2}=4 \frac{d x^{2}+d y^{2}}{\left(1-x^{2}-y^{2}\right)^{2}} .
$$

It is well known that for each regular triangle in the Lobachevsky plane the circumscribed circle exists. The radii emitted out of the center of the circle to the vertices of the triangle forms the angles of $120^{\circ}$.

Let $r$ be the radius of the circumscribed circle. The cosine rule implies that the length $a$ of the side of the regular triangle can be calculated as follows:

$$
\cosh a=\cosh ^{2} r-\sinh ^{2} r \cos \frac{2 \pi}{3}=1+\frac{3}{2} \sinh ^{2} r .
$$

It is easy to verify that for such triangle the length of MST equals $2 a$, and the length of SMT equals $3 r$. Therefore, the Steiner ratio $m(r)$ for the regular triangle inscribed into the circle of radius $r$ in the Lobachevsky plane $L^{2}(-1)$ has the form

$$
m(r)=\frac{3}{2} \cdot \frac{r}{\operatorname{arccosh}\left(1+\frac{3}{2} \sinh ^{2}(r)\right)} .
$$

It is easy to calculate that limit of the function $m(r)$ as $r \rightarrow \infty$ is equal to $3 / 4$. The proof is complete.

Proof of Theorem 1.4. It is easy to see that the Taylor series for the function $m(r)$ at $r=0$ has the following form:

$$
\frac{\sqrt{3}}{2}-\frac{r^{2}}{16 \sqrt{3}}+O\left(r^{4}\right)
$$

Therefore, $m(r)$ is strictly less than $\sqrt{3} / 2$ in some interval $(0, \varepsilon)$. The latter means that for sufficiently small regular triangles on the surfaces of constant curvature -1 , the relation of the lengths of SMT and MST is strictly less than $\sqrt{3} / 2$. The proof is complete.

\section{References}

[1] D. Cieslik, Steiner minimal trees. - Dordrecht, Boston, London, Kluwer Academic Publishers, 1998.

[2] D. Z. Du and F. K. Hwang, A proof of Gilbert-Pollak Conjecture on the Steiner ratio. - Algorithmica, v. 7 (1992) pp. 121-135.

[3] E.N. Gilbert and H.O. Pollak, Steiner Minimal Trees. SIAM J. Appl. Math., 16:1-29, 1968. 
[4] F. K. Hwang, D. Richards, and P. Winter, The Steiner Tree Problem. Elsevier Science Publishers, 1992.

[5] A. O. Ivanov and A. A. Tuzhilin, Minimal Networks. The Steiner Problem and Its Generalizations. - N.W., Boca Raton, Florida, CRC Press, 1994.

[6] A. O. Ivanov and A. A. Tuzhilin, Branching Solutions of One-Dimensional Variational Problems. - World Publisher Press, 2000, to appear.

[7] J. H. Rubinstein and J. F. Weng, Compression theorems and Steiner ratios on spheres. - J. Combin. Optimization, v. 1 (1997) pp. 67-78. 